\title{
Alterations in Activation Gating of Single Shaker A-Type Potassium Channels by the $S^{5}{ }^{5}$ Mutation
}

\author{
William N. Zagotta and Richard W. Aldrich \\ Department of Neurobiology, Stanford University School of Medicine, Stanford, California 94305
}

\begin{abstract}
Alterations in voltage-dependent gating of A-type potassium channels by the $S h^{5}$ mutation were studied using macroscopic and single-channel recording techniques on cultured embryonic myotubes from Drosophila. The mutation increases the voltage required to activate and inactivate the $A_{1}$ channel by approximately $20 \mathrm{mV}$ and decreases the steepness of the voltage dependence of steady-state inactivation. Boltzmann fits to the prepulse inactivation curves have slopes of $e$-fold $/ 3.5 \mathrm{mV}$ for wild type and e-fold $/ 5.0 \mathrm{mV}$ for $S h^{5}$. A kinetic analysis of single $S h^{5} A_{1}$ channels revealed that the transition rates into and out of the open state are not significantly affected by the mutation. In contrast, the latencies until the channel opens following a voltage step are increased at low voltages. These alterations probably do not result from an offset of the transmembrane voltage sensed by the channel as might be expected for an alteration in the surface charge of the protein. By using a kinetic model developed for wild-type $A_{1}$ channels, we show that an alteration in the amplitude and voltage dependence of the deactivation rate for each subunit $(\beta)$ can account for all of the alterations observed.
\end{abstract}

Physiological experiments have provided many insights into the molecular nature of the gating mechanisms of ion channels. cDNA clones for voltage-dependent channels provide a means to test and further refine theories for gating mechanisms and to identify the protein structures involved. A-type potassium channels in Drosophila muscle are coded at least in part by the Shaker gene. Mutations in the gene alter transmission at larval neuromuscular junctions (Jan et al., 1977) and eliminate, reduce, or alter A-type currents in embryonic, larval, and adult muscle (Salkoff and Wyman, 1981b; Wu et al., 1983; Zagotta et al., 1988). This A-channel has been called $A_{1}$ to distinguish it from a second A-type channel found in Drosophila neurons, called $A_{2}$, which differs in single-channel conductance, voltage dependence, kinetics, and is unaffected by mutations of the Shaker locus (Solc et al., 1987; Solc and Aldrich, 1988). Molecular cloning of the Shaker gene (Baumann et al., 1987; Kamb et al., 1987; Papazian et al., 1987; Tempel et al., 1987) has revealed similarity in the derived amino acid sequence with other voltage-gated channels that have been cloned; sodium channels from

\footnotetext{
Received Sept. 21, 1989; revised Dec. 21, 1989; accepted Jan. 4, 1990.

We thank Drs. M. Gautam and M. A. Tanouye for allowing us to discuss their unpublished observations and Drs. D. Baylor and T. Hoshi for helpful comments on the manuscript. This work was supported by U.S. Public Health Service grant NS 23294 to R.W.A. and Training Grant NS 07158 to W.N.Z.

Correspondence should be addressed to Richard W. Aldrich at the above address.

Copyright (C) 1990 Society for Neuroscience $0270-6474 / 90 / 061799-12 \$ 02.00 / 0$
}

eel electric organ (Noda et al., 1984), rat brain (Noda et al., 1986; Kayano et al., 1988), and Drosophila (Salkoff et al., 1987, Loughney et al., 1989), and calcium channels from vertebrate skeletal muscle (Tanabe et al., 1987, 1988; Ellis et al., 1988). An intriguing finding from the cloning of the $S h$ gene is that the locus is extensively alternatively spliced, with the potential for as many as 24 different species of mRNA produced (Kamb et al., 1988; Pongs et al., 1988; Schwarz et al., 1988) The deduced proteins of these transcripts share a central core region containing most of the putative transmembrane segments and differ in the putative cytoplasmic amino and carboxyl regions. Oocytes that have been injected singly with different $S h$ mRNA species produce A-type currents with similar voltage dependence but different kinetics, differing primarily in the rate of inactivation and recovery from inactivation (Timpe et al., 1988a, b; Iverson et al., 1988; Zagotta et al., 1989a). These functional alterations in variants with known structural differences provide a starting point for studies of the role of particular residues in gating.

Voltage-gated channels must have charged structures in the membrane electric field that move in response to changes in voltage. The movement of these charges in the voltage-dependent sodium channel and Shaker $(S h)$ A-type potassium channel is associated with activation transitions of the channels, while the inactivation transition does not produce significant charge movement across the membrane field (Armstrong and Bezanilla, 1977; Aldrich et al., 1983; Aldrich and Stevens, 1987; Zagotta and Aldrich, 1989; Zagotta et al., 1989a). The $S h$ potassium channel shares, with most other cloned voltage-gated channels, an amphipathic region, called $\mathrm{S} 4$, with a recurring motif of a positively charged amino acid at every third position with intervening hydrophobic residues (Noda et al., 1984, 1986; Salkoff et al., 1987; Tanabe et al., 1987, 1988; Tempel et al., 1987, 1988; Baumann et al., 1988; Ellis et al., 1988; Kayano et al., 1988). The $\mathrm{S} 4$ region has been postulated to span the membrane and act as the voltage-dependent gating charge for these channels (Greenblatt et al., 1985; Catterall, 1986; Guy and Seetharamulu, 1986; Noda et al., 1986). Stiuhmer et al. (1989) have shown that mutations of the charged residues in the $\mathrm{S} 4$ of the first homology domain of the sodium channel are associated with alterations of the channel's voltage dependence in a manner consistent with its theorized role.

While site-directed mutagenesis studies are proving useful for testing specific theories and defining the role of particular regions of channel proteins, they are unlikely to identify residues or small regions with unexpected roles in channel function. To identify these regions, a genetic approach may be more useful. By applying mutagens and a selection criterion, physiologically interesting mutations can be identified in a channel structural 

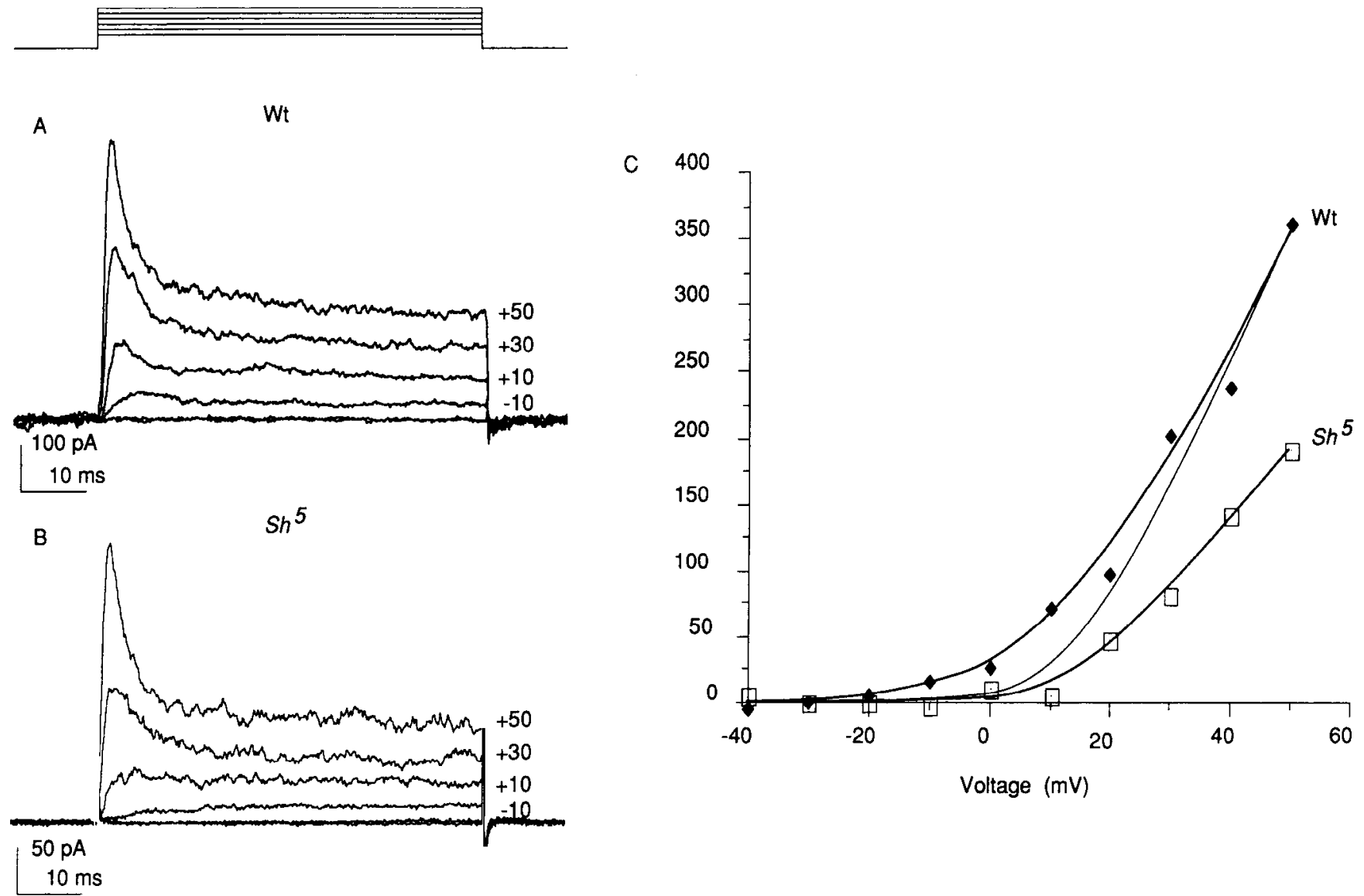

Figure 1. Comparison of voltage dependence of activation of wild-type and $S h^{5} \mathrm{~A}_{1}$ channels. Whole-cell current families in wild-type $(A)$ and $S h^{5}$ mutant $(B)$ myotubes. The membrane voltage was stepped from a holding voltage of $-70 \mathrm{mV}$ to a prepulse voltage of $-100 \mathrm{mV}$ for $500 \mathrm{msec}$ and then depolarized for $60 \mathrm{msec}$ to command voltages between -50 and $+50 \mathrm{mV}$ in $20 \mathrm{mV}$ increments. The voltage pulse protocol is shown at the top. The data were filtered at $2 \mathrm{kHz}$ and digitized at $100 \mu \mathrm{sec} /$ point $C$, Peak $I(V)$ curve for the whole-cell A-currents. The peak A-current was calculated by subtracting the current $50 \mathrm{msec}$ after the start of the pulse from the current at the peak of the outward currents. The smooth curves were drawn by eye. The thin line shows the curve fitted to the $S h^{5}$ data scaled to equal the wild-type data at $+50 \mathrm{mV}$.

gene. The molecular nature of the mutation can then be determined by sequence analysis.

One such physiologically interesting mutation already exists for the $S h \mathrm{~A}_{1}$ potassium channel in Drosophila. The $S h^{5} \mathrm{mu}-$ tation was induced by the chemical mutagen ethyl methanesulfonate (EMS) and identified on the basis of leg shaking under ether anesthesia (Kaplan and Trout, 1969). $S h^{5}$ was subsequently shown, by 2-electrode voltage-clamp of larval and pupal muscle, to alter the voltage-dependent gating of the $S h$ A-current (Salkoff and Wyman, 1981b; Wu and Haugland, 1985). Unlike the differences in gating among alternatively spliced $S h$ variants, the effects of $S h^{5}$ in larval muscle were primarily on the voltage dependence of the A-current. In this article, we examine the alterations in gating of single $\mathrm{A}_{1}$ channels caused by the $S h^{5}$ mutation and interpret them in the context of a kinetic model developed for wild-type $A_{1}$ channels (Zagotta and Aldrich, 1989). The manner in which the gating is altered provides insights into the role of the mutated structure in the gating mechanism.

\section{Materials and Methods}

The $S h^{\varsigma}$ strain was obtained from L. Salkoff of Washington University (St. Louis, MO). The cell culture and electrophysiological method have been described previously (Zagotta and Aldrich, 1989).

Solutions. For whole-cell and outside-out experiments the composi- tions of the solutions were as follows (in mM): external (bath) $140 \mathrm{NaCl}$, $2 \mathrm{KCl}, 6 \mathrm{MgCl}_{2}, 5 \mathrm{HEPES}, \mathrm{pH} \mathrm{7.1}$; and internal (pipet) $140 \mathrm{KCl}, 2$ $\mathrm{MgCl}_{2}, 11$ EGTA, 10 HEPES, pH 7.1. For some whole-cell and outsideout experiments, internal $140 \mathrm{mM} \mathrm{KCl}$ was replaced by $70 \mathrm{~mm} \mathrm{KCl}$ and $70 \mathrm{~mm} \mathrm{KF}$, and external $6 \mathrm{mM} \mathrm{MgCl}$, was replaced by $4 \mathrm{~mm} \mathrm{MgCl}$, and $2 \mathrm{mM} \mathrm{CaCl}_{2}$. Under these conditions, no marked differences in the kinetics or voltage dependence of the A-current were observed. For cellattached experiments, the external solution was used in the pipet and the internal, high- $\mathrm{K}^{+}$solution was used in the bath. In this high- $\mathrm{K}^{+}$ solution, the cell's resting potential should be close to $0 \mathrm{mV}$ and therefore the voltages reported are close to the actual voltage across the membrane. No obvious differences were seen in the voltage dependence for any of the channels between cell-attached and cell-free patches, although there was a tendency for channels in cell-free patches to open more times during a voltage step. The free $\mathrm{Ca}^{2+}$ concentration of the internal solution was estimated to be approximately $1-10 \mathrm{nM}$.

The temperature of the recording chamber was controlled at $22 \pm$ $0.2^{\circ} \mathrm{C}$.

\section{Results}

Macroscopic A-type potassium currents affected by mutations of the $S h$ locus have been examined under voltage-clamp in 3 different preparations, pupal (or adult) dorsolongitudinal flight muscles (DLMs) (Salkoff and Wyman, 1981a, b, 1983; Salkoff, 1983a, b; Timpe and Jan, 1987), larval body wall muscles (Wu et al., 1983; Wu and Haugland, 1985), and cultured embryonic myotubes (Solc et al., 1987; Zagotta et al., 1988). While many 

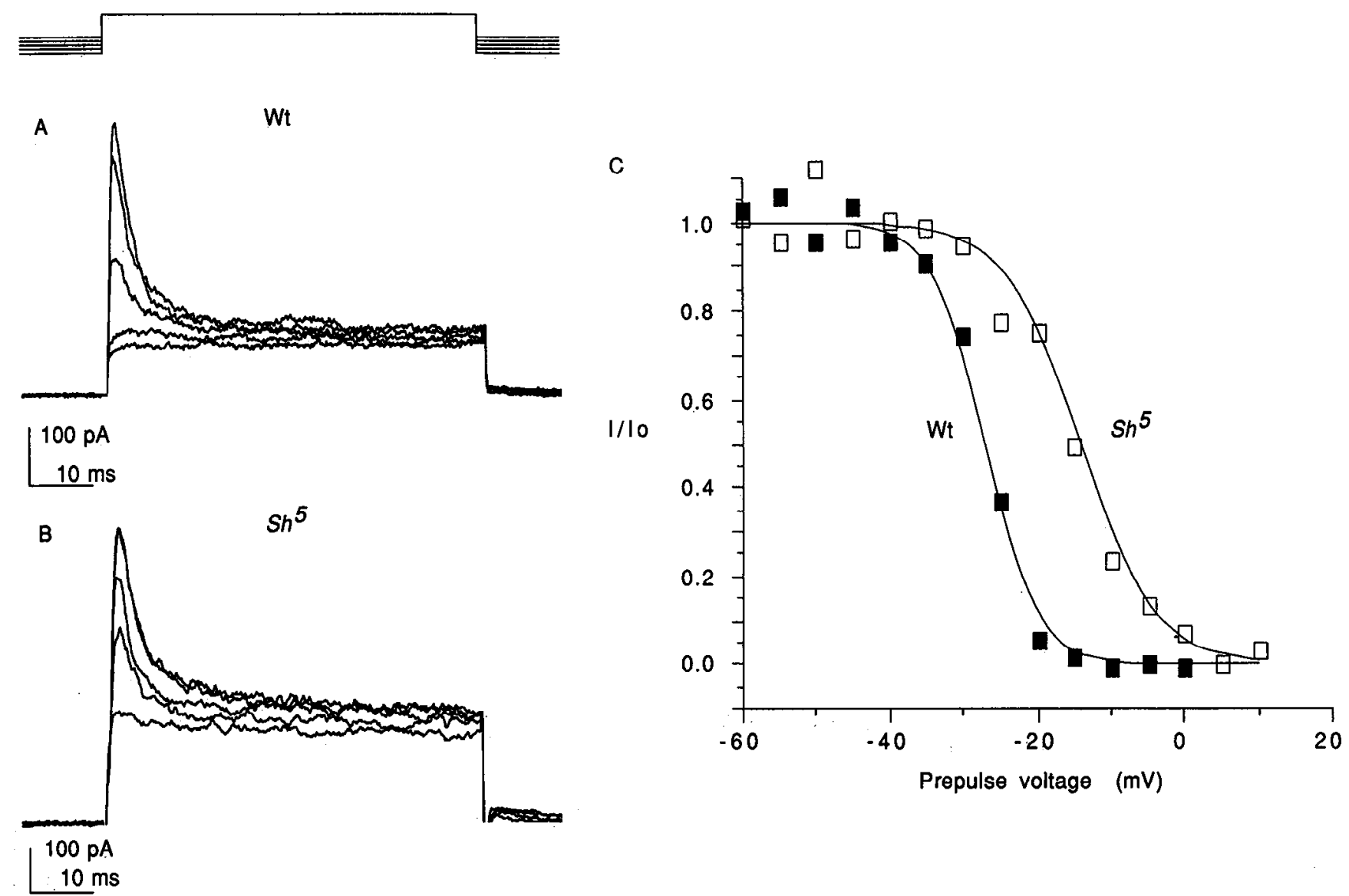

Prepulse voltage $(\mathrm{mV})$

Figure 2. Comparison of voltage dependence of prepulse inactivation of wild-type and $S h^{5} \mathrm{~A}$, channels. Whole-cell current families in wild-type $(A)$ and $S h^{5}$ mutant $(B)$ myotubes. The whole-cell currents were elicited by steps to $+50 \mathrm{mV}$ following a 500 msec prepulse to $-45,-35,-25$, -15 , and $-5 \mathrm{mV}$ from a holding voltage of $-70 \mathrm{mV}$. The voltage pulse protocol is shown at the top. The data were filtered at $2 \mathrm{kHz}$ and digitized at $100 \mu \mathrm{sec} /$ point. $C$. Prepulse inactivation curve for the whole-cell A-currents. Normalized peak A-current during the step to $+50 \mathrm{mV}$ is plotted versus prepulse voltage. The normalized A-current was calculated by subtracting the current $50 \mathrm{msec}$ after the start of the pulse from the current at the time of the peak of the large transient currents and normalizing to 1 at large hyperpolarized voltages. The different symbols represent data from 7 different myotubes. The data are fitted by a Boltzmann distribution (Eq. 1) with a slope factor $(Y)$ of $3.5 \mathrm{mV}$ and a half-inactivation voltage $\left(V_{1 / 2}\right)$ of $-27 \mathrm{mV}$ for wild-type and a slope factor of $5.0 \mathrm{mV}$ and a half-inactivation voltage of $-16 \mathrm{mV}$ for $S h^{5}$.

mutations of the $S h$ locus completely eliminate A-type potassium currents in these muscle preparations, the $S h^{s}$ allele alters the voltage-dependent kinetics of the A-current (Salkoff and Wyman, 1981 b; Wu and Haugland, 1985). The alterations of the A-current in myotubes are illustrated in Figure 1. The outward currents were recorded using the whole-cell configuration of the patch-clamp technique on myotubes 10-14 h after plating, when the A-type current represents a significant portion of the total outward current (Zagotta et al., 1988). Figure $1 A$ shows the currents from a wild-type myotube for comparison, and Figure $1 B$ shows currents from a myotube homozygous for the $\mathrm{Sh}^{5}$ mutation. The effects of $\mathrm{Sh}^{5}$ can be seen qualitatively in these current families. At the low voltages, the $S h^{s}$ outward currents have a slower macroscopic activation and inactivation rate. The difference in voltage dependence can also be seen in the $I(V)$ curves in Figure 1C. To reduce the amount of contamination by delayed potassium currents in these $I(V)$ curves, the peak current minus the steady-state current (at $50 \mathrm{msec}$ ) is plotted versus the test voltage. The $S h^{5} I(V)$ curve lies to the right of the wild-type curve. Scaling the $S h^{5}$ data to the wild-type peak current at $+50 \mathrm{mV}$ illustrates that the differences in the $I(V)$ curves are not simply the result of differences in the number of channels between these 2 particular cells. While the absolute magnitude of the current is variable from cell to cell in both wild-type and $S h^{5}$, the A-currents in $S h^{5}$ myotubes consistently required more depolarized voltages to activate $(N=10)$.

In addition to activating at more depolarized voltages, the A-current in $S h^{5}$ myotubes requires more depolarized voltages to inactivate. Figure 2 shows families of currents elicited by test pulses to $+50 \mathrm{mV}$ following $500 \mathrm{msec}$ prepulses to $-45,-35$, $-25,-15$, and $-5 \mathrm{mV}$. While the wild-type $A_{1}$ channels (Fig. $2 A$ ) are more than half inactivated by prepulses to $-25 \mathrm{mV}$, only $25 \%$ of the $S h^{s}$ channels (Fig. $2 B$ ) are inactivated at that voltage. Figure $2 C$ plots an estimate of the fraction of channels available to open (not inactivated) versus the prepulse voltage. The estimate was calculated by subtracting the current at 50 msec after the start of the test pulse from the current at the time of the peak of the large transient currents and normalizing the asymptote to 1 at large hyperpolarized prepulses. The points were fitted by eye with a Boltzmann distribution:

$$
P(V)=\frac{I}{I_{\max }}=\frac{1}{1+\exp \left[\left(V-V_{y / 2} / y\right]\right.}
$$

The Boltzmann distribution fitted to the wild-type prepulse inactivation data has a midpoint of inactivation $\left(V_{1 / 2}\right)$ of $-27 \mathrm{mV}$ 
A

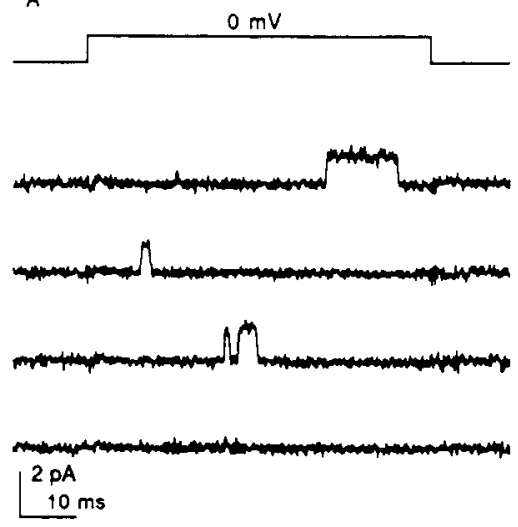

B
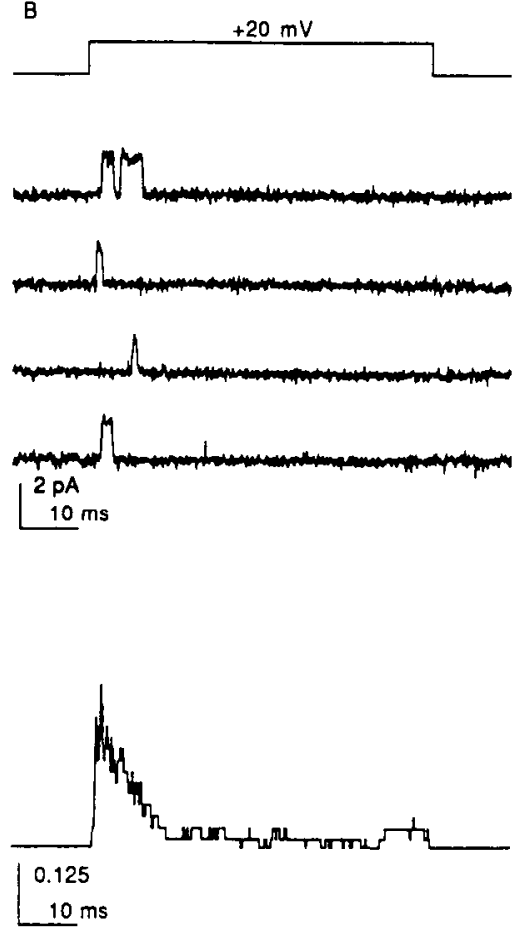

$c$
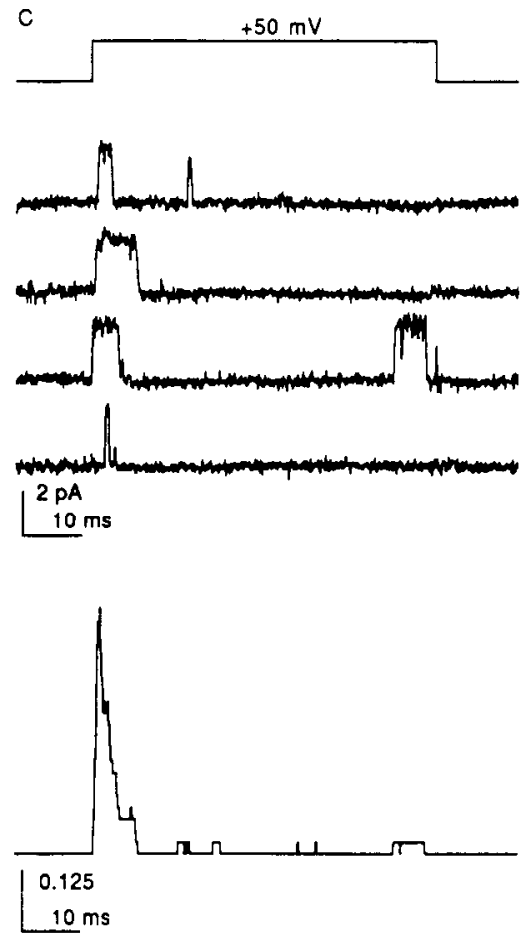

Figure 3. Representative openings of a single $S h^{5} \mathrm{~A}_{1}$ channel at $0 \mathrm{mV}(A),+20 \mathrm{mV}(B)$, and $+50 \mathrm{mV}(C)$. The channels were recorded from an outside-out patch containing a single $A_{1}$ channel. The voltage protocol is shown at the top. The data were filtered at $2 \mathrm{kHz}$ and digitized at 50 $\mu \mathrm{sec} /$ point.

and a slope factor $(Y)$ of $e$-fold for $3.5 \mathrm{mV}$, while that for the $S h^{5}$ data has a midpoint of $-16 \mathrm{mV}$ and a slope factor of $e$-fold for $5.0 \mathrm{mV}$. These alterations in midpoint and slope of the prepulse inactivation curve of $S h^{5}$ A-current were a consistent feature in every cell examined $(N=14)$ and can be seen for 6 cells in Figure $10 B$. The alterations in $I(V)$ and prepulse inactivation curves produced by $S h^{5}$ are similar to the effects seen on A-currents in larval muscle (Wu and Haugland, 1985).

To determine the alterations in transition rates between kinetic states that underlie the differences in macroscopic voltage dependence and kinetics, we recorded and analyzed the currents through single $\mathrm{A}_{1}$ channels in $S h^{5}$ myotubes. Examples of single $S h^{5} A_{1}$ channel records at $0,+20$, and $+50 \mathrm{mV}$ from an outsideout patch and their corresponding ensemble a verages as shown in Figure 3. The general behavior of the $S h^{5}$ channels is similar to wild-type $A_{1}$ channels (Zagotta et al., 1988; Zagotta and Aldrich, 1989). The channels open in single events or in short bursts of 2 or 3 openings separated by short-lived closed events, and inactivate by transitions into long-lived closed states. Occasionally, a channel will return from inactivation and burst a second time later in the pulse. These records also illustrate the voltage dependence in the time between the onset of the voltage pulsc and the first opening, the first latency, but not in the transitions occurring after opening (Zagotta and Aldrich, 1989; Zagotta et al., 1989a). Like the wild-type $\mathrm{A}_{1}$ channels, these channels occur relatively infrequently in patches and usually disappear after only a few minutes. Channel openings in cellattached patches also tend to disappear shortly after seal formation, thus rundown of the native channel is not limited to patch configurations where membrane contact with the cytoplasm has been disrupted. A similar rundown process has been reported for the $\mathrm{ShA}$ or $\mathrm{ShC}$ alternatively spliced variants ex- pressed in Xenopus oocytes (Timpe et al., 1988a,b) but is not present when ShB channels are expressed in Drosophila myotubes by germline transformation (Zagotta et al., 1989b). The rundown is likely, therefore, to be a property of the $S h$ variant(s) comprising the native channel and to result from a very slow recovery from a slow inactivation process related to opening of the channels or to depolarization. This slow inactivation process is slow compared to the fast gating processes considered here and therefore will be disregarded in the kinetic model as it was for the wild-type model. While our experiments on $S h^{5}$ single channels were limited by this behavior, they were facilitated by the high probability of obtaining patches with only a single channel.

Figure $4 A, B$, shows histograms of the open durations at 0 and $+50 \mathrm{mV}$, respectively. Like the open-duration histograms shown previously for the wild-type $A_{1}$ channel (Zagotta and Aldrich, 1989, Fig. 5), these histograms are well described by a single exponential distribution with a time constant of $1.5 \mathrm{msec}$. The lack of any appreciable voltage dependence in the mean open durations and their similarity to the mean open durations of the wild-type channel are shown in Figure $4 C$. The vertical bars in this plot illustrate the range of the mean open durations shown previously for the wild-type channel at each voltage ( $\mathrm{Za}$ gotta and Aldrich, 1989). The different symbols represent the mean open durations for 5 different $S h^{5} \mathrm{~A}_{1}$ channels from singlechannel patches.

The bursts of the $S h^{5} \mathrm{~A}_{1}$ channels were not distinguishable from wild type. Figure $5 \mathrm{~A}$ plots the voltage dependence of the probability of terminating a burst for 5 different $S h^{5}$ channels, along with the ranges for the wild-type channel at each voltage. As for the wild-type channel, the burst is considered to be terminated by closed events longer than $1 \mathrm{msec}, 3-4$ times the fast 


\section{Open Durations}

A
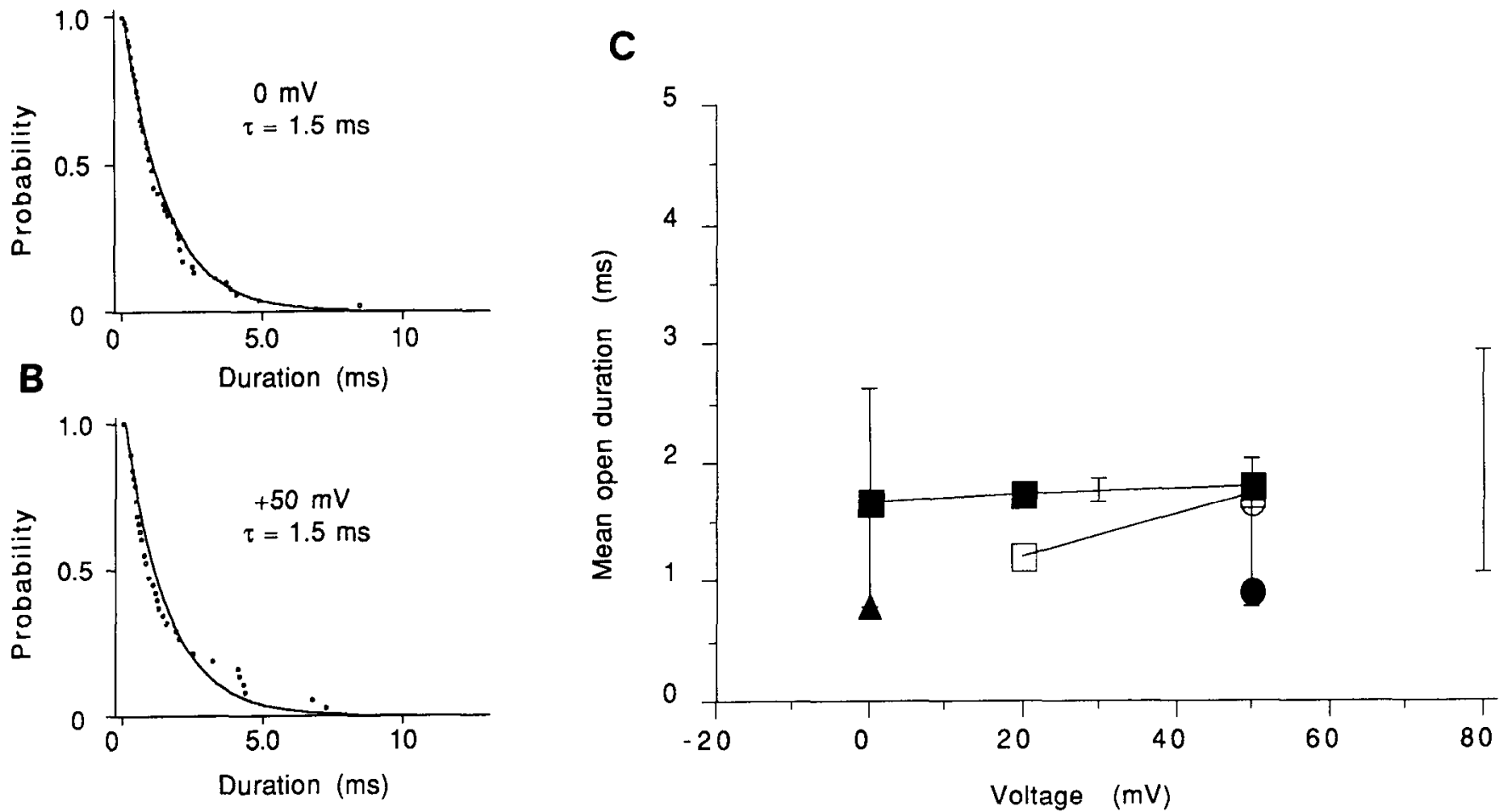

Figure 4. Comparison of voltage dependence of open durations of wild-type and $S h^{5} \mathrm{~A}_{1}$ channels. $A$, Tail distribution of open durations of a $S h$ $A_{1}$ channcl during voltage steps to $0 \mathrm{mV}$. Tail distributions describc the probability of an opcning being longer than the duration on the abscissa and are calculated as one minus the cumulative distribution. The histogram is fitted by a single-exponential function with a time constant of 1.5 msec. $B$. Tail distribution of open durations of a $S h^{5} \mathrm{~A}_{1}$ channel during voltage steps to $+50 \mathrm{mV}$, fitted by a single-exponential function with a time constant of $1.5 \mathrm{msec}$. $C$. The mean open duration of $5 S h^{5} \mathrm{~A}_{1}$ channels is plotted versus the step voltage. The vertical bars in this plot illustrate the range of mean open durations of wild-type channels at each voltage (Zagotta and Aldrich, 1989).

component of the closed-duration histograms. The probability of burst termination $(q)$ was determined by fitting histograms of the number of openings per burst $(n)$ with a geometric distribution:

$$
P(n)=(1-q)^{(n-1)} q \text { for }(n=1,2,3 \ldots)
$$

This probability $(q)$ for the $S h^{s}$ channel showed no appreciable or consistent voltage dependence and nearly always fell within the range of the wild-type channels. Together with the openduration data, these results suggest the channel assumes a single open state with voltage-independent leaving rates similar to the wild-type channel. Figure $5 B$ plots the voltage dependence of the mean closed durations within a burst for 5 different $S h^{5}$ channels along with the wild-type ranges. The similarity in burst closed durations between wild-type and $S h^{5}$ channels suggests that the rate entering the open state from the burst closed state is also not significantly altered by the mutation.

Table 1 summarizes the single-channel gating parameters so far discussed between wild-type and $S h^{s} \mathrm{~A}_{1}$ channels. The similarity of these parameters indicates that the gating transitions occurring after the channel first opens are not significantly affected by the $S h^{5}$ mutation and that the altered gating is likely to result from an effect on the transitions occurring before the channel opens. To further illustrate this point, we have separated the kinetic transitions of the channel into 2 classes: (1) transitions that occur from the onset of the voltage pulse until the channel opens for the first time, and (2) transitions that occur after the channel opens for the first time. Some transitions, such as the final opening transition of the activation pathway, may belong to both classes. The first latency probability density function, $f(t)$, contains contributions only from transitions in the first class. Conversely, a second function, $m(t)$, describing the conditional probability of a channel being open at time $t$ given that it opened at $t=0$, contains contributions only from transitions in the second class. $f(t)$ and $m(t)$ are related to the prob-

Table 1. Comparison of single-channel parameters between wild-type and $S h^{5} A_{1}$ channels

\begin{tabular}{lrrr} 
Parameter & $\begin{array}{l}\text { Volt- } \\
\text { age } \\
(\mathrm{mV})\end{array}$ & \multicolumn{1}{l}{ Wild-type } & \multicolumn{1}{l}{$S h^{5}$} \\
\hline Open duration & 50 & $1.46 \pm 0.41(8)$ & $1.52 \pm 0.41(4)$ \\
(msec) & 0 & $1.58 \pm 0.71(6)$ & $1.24 \pm 0.62(2)$ \\
Probability of burst & 50 & $0.7 \pm 0.15(8)$ & $0.8 \pm 0.10(4)$ \\
$\quad$ termination & 0 & $0.7 \pm 0.14(6)$ & $0.6 \pm 0.13(3)$ \\
Burst closed duration & 50 & $0.25 \pm 0.03(7)$ & $0.30 \pm 0.04(4)$ \\
$\quad$ (msec) & 0 & $0.25 \pm 0.04(6)$ & $0.25(1)$
\end{tabular}

Each parameter is expressed by a mean \pm SD for the number of single-channel patches indicated in parentheses. 
A

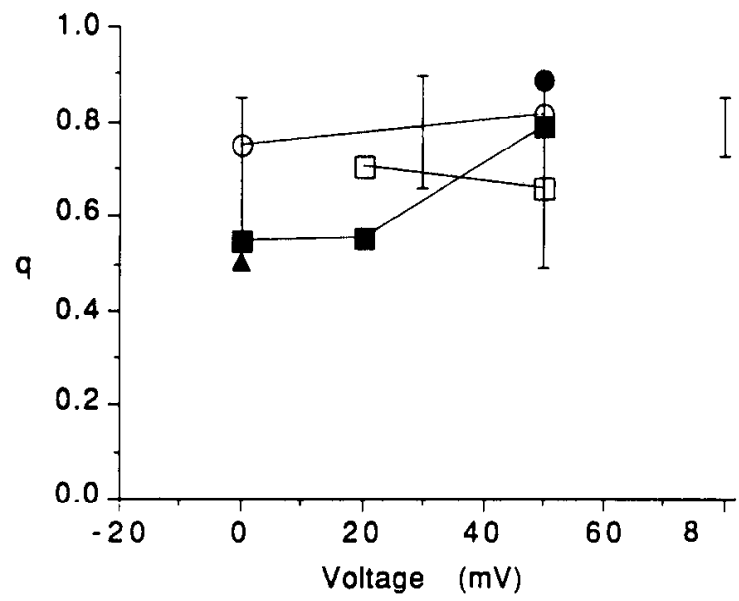

B

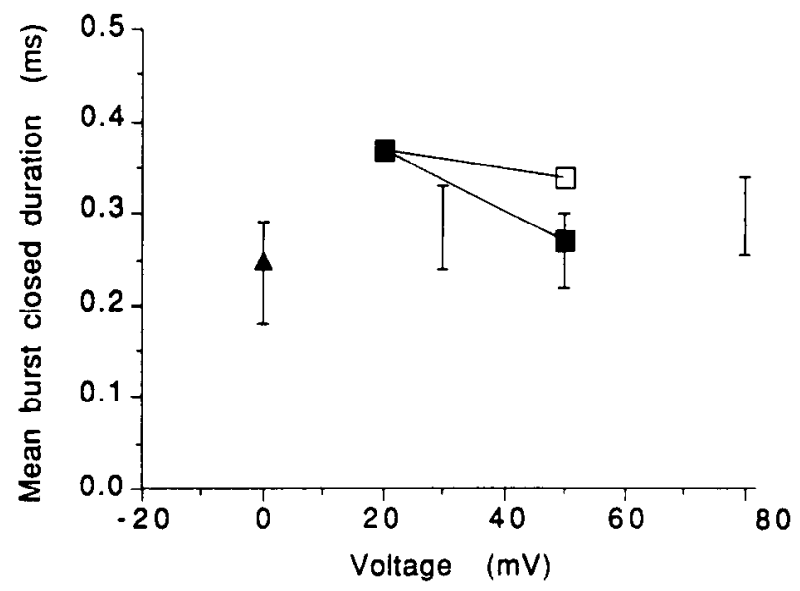

Figure 5. Comparison of voltage dependence of burst parameters of wild-type and $S h^{5} \mathrm{~A}_{1}$ channels. $A$, Voltage dependence of the probability of tcrminating a burst. The probability of terminating a burst $(q)$ of $5 S h^{5} \mathbf{A}_{1}$ channels is plotted versus the step voltage. The vertical bars illustrate the range of $q$ of wild-type channels at each voltage. $B$. Voltage dependence of burst closed durations. The mean burst closed duration of $5 S h^{5} A_{1}$ channels is plotted versus the step voltage. The vertical bars illustrate the range of mean burst closed durations of wild-type channels at each voltage (Zagotta and Aldrich, 1989).

ability of the channel being open after a voltage step $P(t)$ by the following convolution integral:

$$
P(t)=\int_{0}^{t} f(t-\tau) m(\tau) d \tau
$$

We have shown previously for wild-type $A_{1}$ channels, and $S h$ channels in Xenopus oocytes, that the voltage dependence of $P(t)$ arises almost exclusively from the voltage dependence of $f(t)$ (Zagotta et al., 1989a).

To investigate whether $S h^{5}$ alters $m(t)$ or $f(t)$, we have compared these distributions for the wild-type and $S h^{5} \mathrm{~A}_{1}$ channels. As shown in Figure 6, the conditional probability functions, $m(t)$, for the 2 genotypes nearly superimpose for data at both 0 and $+50 \mathrm{mV}$. In contrast, the first latency function, $f(t)$, is alter- ed by the mutation, suggesting that the primary difference between wild-type and $S h^{5} \mathrm{~A}_{1}$ channels lies in the activation pathway. Figure 7 shows the first latency functions for a $S h^{s} A_{1}$ channel at $0,+20$, and $+50 \mathrm{mV}$ in their integral form as distribution functions. The smooth curves plotted with the $S h^{5}$ latency distributions are latency distributions at the same 3 voltages calculated from the model of Zagotta and Aldrich (1989) that fits the wild-type data. As for wild-type latencies, the $S h^{5}$ first latencies are highly voltage-dependent. However, especially at the lower voltages, the latencies are longer for the $S h^{5} \mathrm{~A}_{1}$ channel than for the wild-type channel.

One possible way to explain the increased depolarization needed to activate and inactivate $S h^{5}$ A-current is if the mutation caused simply a shift in the voltage dependence of the A-current. This could result from an alteration in the trans-
A

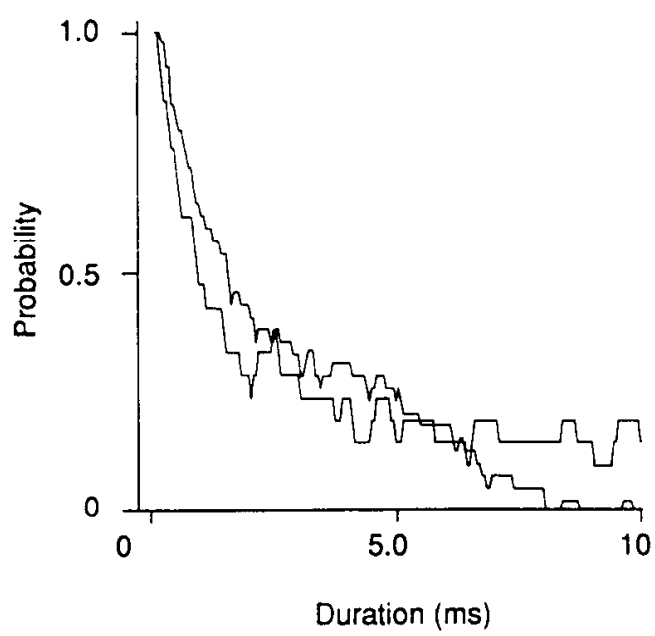

B

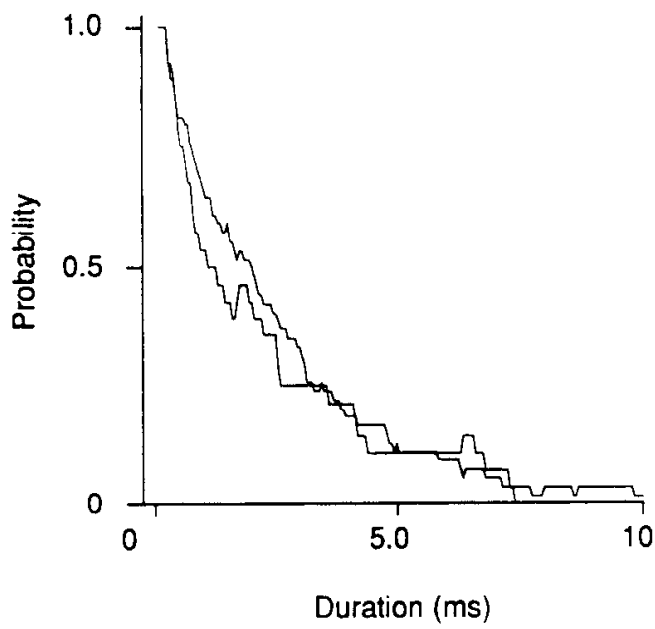

Figure 6. Comparison of conditional probability functions, $m(t)$, of wild-type and $S h^{5} \mathrm{~A}_{1}$ channels at $0 \mathrm{mV}(A)$ and $+50 \mathrm{mV}(B)$. The function $m(t)$ represents the probability that the channel was open at time $t$ given that it first opened at $t=0$. The distributions were calculated by synchronizing all records at the time of the first opening and averaging them. Each of the patches shown contained only one active channel. 
membrane voltage sensed by the channel molecule caused by a change in membrane surface potential. An offset in the transmembrane voltage of $20 \mathrm{mV}$ would predict that currents from wild-type myotubes should be identical to currents from $S h^{5}$ myotubes recorded at $20 \mathrm{mV}$ higher voltages. To explore this possibility we overlaid the macroscopic potassium currents from wild-type and $S h^{5}$ myotubes. In Figure $8 A$ the currents from corresponding voltage pulses are overlaid and the currents scaled so that the peak currents at $50 \mathrm{mV}$ coincide, thus eliminating influences from differences in total number of channels between these 2 cells. As pointed out in Figure 1, at lower voltages the wild-type current inactivates much more rapidly than the $S h^{5}$ current, and its peak represents a larger fraction of its value at $50 \mathrm{mV}$. However, at $50 \mathrm{mV}$ the A-currents from wild-type and $S h^{5}$ have nearly identical macroscopic activation and inactivation kinetics.

In contrast, when the currents are overlaid from voltages different by $20 \mathrm{mV}$ (Fig. $8 B$ ) the converse is true. The currents overlay well at the lower voltages, but the wild-type current at $30 \mathrm{mV}$ is considerably slower than the $S h^{5}$ current at $50 \mathrm{mV}$ (top traces). While more depolarized voltages are needed in $S h^{5}$ to produce currents with similar amplitude and kinetics as wildtype at low voltages, at high voltages $(50 \mathrm{mV})$ the currents are similar. This is inconsistent with a simple shift in voltage dependence and suggests a steepening of the peak conductancevoltage relation of $S h^{5} \mathrm{~A}_{1}$ channels. While we cannot totally eliminate the differential contribution of the delayed currents when noncorresponding voltages are overlaid, it seems that the effects of $S h^{5}$ are not simply a voltage shift.

Figure 9 compares the voltage dependence of $S h^{5}$ A-current with the predictions of a voltage-offset model for the $S h^{5}$ alterations. The normalized peak conductance-voltage relations, $G(V)$ curves, for $4 S h^{5}$ myotubes are plotted in Figure $9 A$. The $G(V)$ curves were determined by dividing $I(V)$ curves like in Figure $1 C$ by the unitary current through single $A_{1}$ channels and normalizing the asymptote to 1 at high voltages. The smooth curves superimposed on the data are the $G(V)$ curves from the model fitting the wild-type data in Zagotta and Aldrich (1989) with and without a $20 \mathrm{mV}$ depolarizing shift. This shifted curve represents the expected $G(V)$ curve arising from an offset of the transmembrane voltage sensed by the wild-type channel since the unitary current voltage relation is linear in these solutions (Zagotta et al., 1988). The expected curve falls within the range of data points at each voltage. However, it has a tendency to overestimate the conductance at low voltages and underestimate it at high voltages, suggesting the possibility that the $S h^{5} G(V)$ curve is slightly steeper than the wild-type curve.

A voltage-offset model also predicts that more depolarized voltages are needed to inactivate the channel, even though the inactivation transition of the channel is voltage-independent. Figure $9 B$ plots the prepulse inactivation data from a number of $S h^{5}$ myotubes together with the unshifted and shifted curves from fits to wild-type data (Zagotta and Aldrich, 1989). The $S h^{5}$ prepulse inactivation curve appears to have a lesser slope than the wild-type curve as pointed out in Figure $2 C$. While we have attempted to eliminate the contribution of delayed currents to these prepulse inactivation data by plotting the peak minus steady-state current, we cannot totally eliminate the possibility that the altered slope is a result of a differential contribution of the delayed current in wild-type and $S h^{5}$ myotubes. However, to the extent that the slope of the prepulse inactivation curve is altered by the $S h^{5}$ mutation, the $S h^{5}$ data is inconsistent with

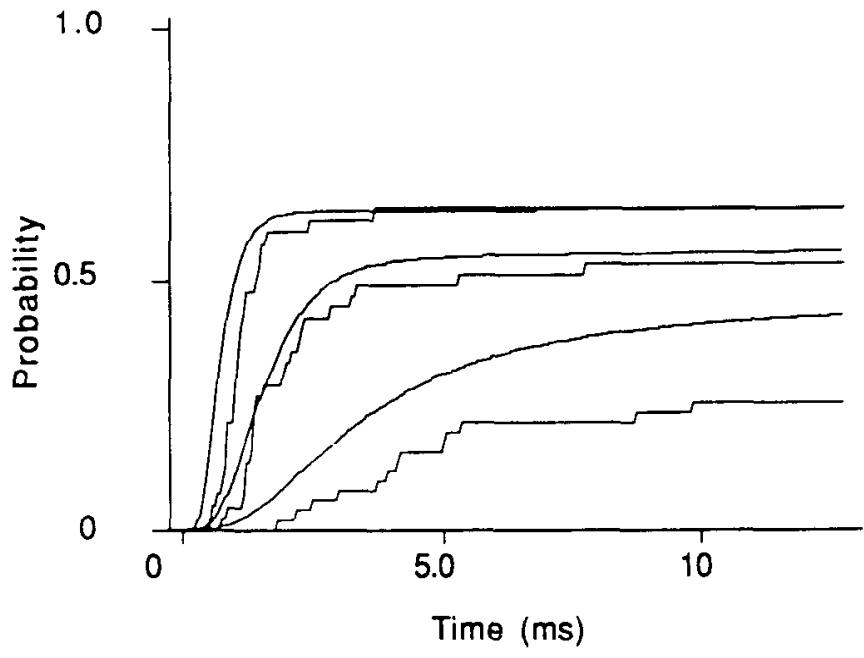

Figure 7. Comparison of voltage dependence of first latencies of wildtype and $S h^{5} \mathrm{~A}_{1}$ channels. Cumulative distributions of first latencies of a $S h^{5} A_{1}$ channel during voltage steps to $0,+20$, and $+50 \mathrm{mV}$ are shown by the rough curves. The smooth curves are first latency distributions at the same 3 voltages from fits to wild-type data (Zagotta and Aldrich, 1989).

the voltage-offset model. Since, for the $A_{1}$ channel, the slope of the prepulse inactivation curve is a better indicator of the amount of charge movement during the activation processes than the $G$ (V) curve (Zagotta and Aldrich, 1989), this result suggests the $S h^{5}$ mutation alters the charge movement, and corresponding voltage dependence, of one or more of the activation transitions. By determining which rates are affected and how, we can begin to postulate what sort of structural changes must occur with the $S h^{5}$ mutation.

To examine the activation rates in $S h^{5}$, we have utilized the model developed for the wild-type $A_{1}$ channel. In short, channel activation was assumed to occur by the independent movement of 4 identical gating charges, perhaps represented by protein subunits. All 4 gating charges must move to allow the channel to open, but the final opening transition is voltage independent. (A summary of the model is shown in Figure 10.) A channel that contains more than one kinetically distinct subunit would require a model with many more closed states in the opening pathway. The movement of each gating charge was assumed to occur at a rate exponentially dependent on voltage. The voltage dependence of the activation rates were therefore constrained by the following equations:

$$
\alpha(V)=A_{\alpha} \exp \left(V / V_{\alpha}\right) \text { and } \beta(V)=A_{\beta} \exp \left[-\left(V / V_{\beta}\right)\right],
$$

where $A_{\alpha}$ and $A_{\beta}$ are the rates at $0 \mathrm{mV}$ of $\alpha$ and $\beta$, respectively, and $V_{\alpha}$ and $V_{\beta}$ specify their voltage dependencies. The inactivation rates $(\kappa$ and $\lambda)$ are independent of voltage. As in the modeling of the wild-type $A_{1}$ channel, to determine the magnitude and voltage dependence of the transition rates for channel activation, model parameters must be found which adequately describe all of the macroscopic and single-channel properties. However, since the open durations and burst parameters for $S h^{5} \mathrm{~A}_{1}$ channels are indistinguishable from wild-type, the transition rates directly into and out of the open state $(\gamma, \delta, \kappa$, and $\lambda$ ) were not changed from wild-type. In addition, the transition rates for inactivation from the closed state are also considered to be unchanged as the occurrence of blank sweeps, while variable, was similar to that of the wild-type channel.

The fitting procedure was similar to that described previously 
A

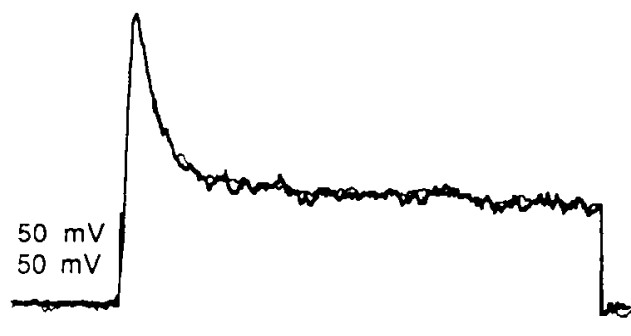

B

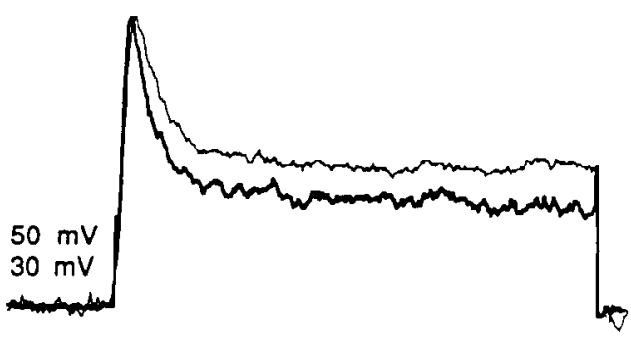

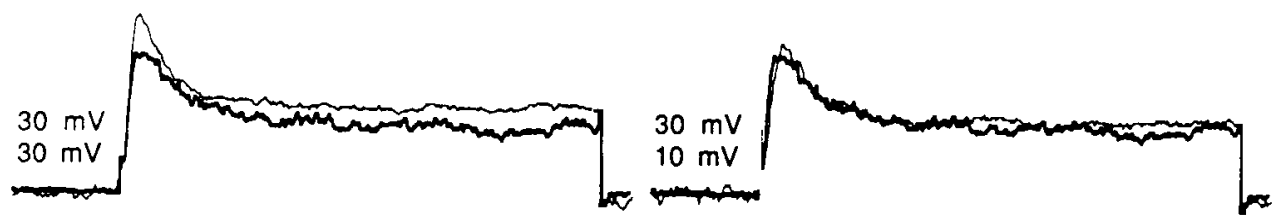

Figure 8. Superposition of whole-cell currents from wild-type and $S h^{5}$ myotubes at corresponding voltages $(A)$ and voltages separated by $20 \mathrm{mV}(B)$. The thinner trace represents data from a wild-type myotube, while the bolder trace is from a $S h$ myotube. The test voltages for each pair of traces are shown to the left of the traces, with the wildtype voltage at the bottom and the $S h^{5}$ voltage at the top. The current families were scaled so that the peak currents at the highest voltage are the same.

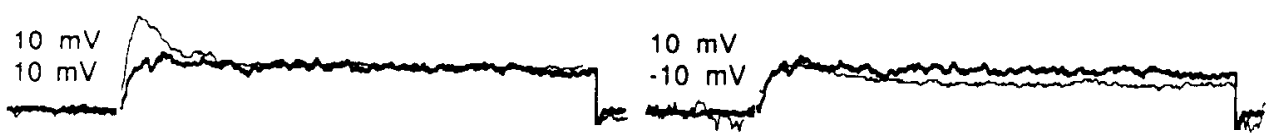

A

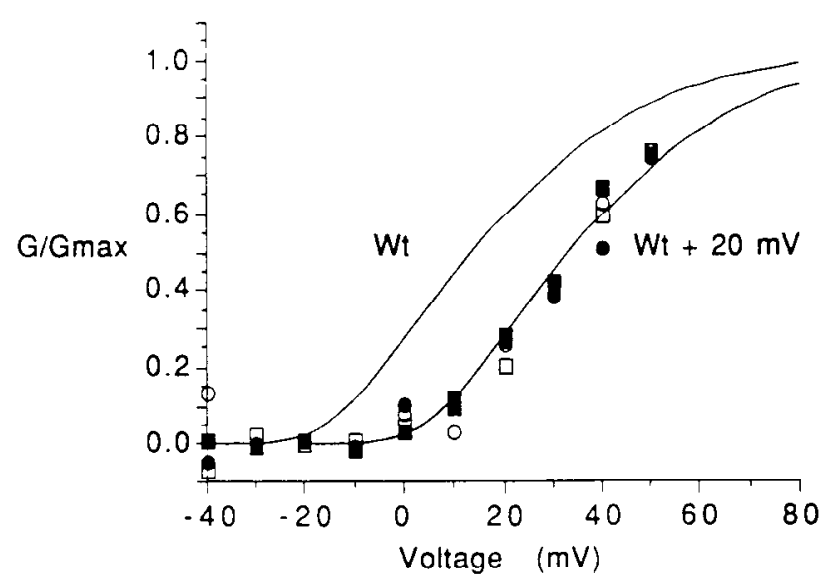

Peak Conductance vs Voltage

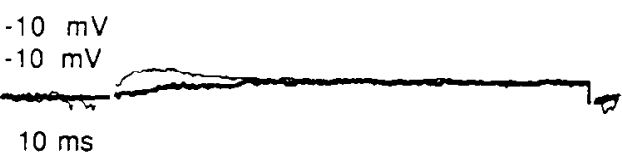

$-10 \mathrm{mV}$

$-30 \mathrm{mV}$

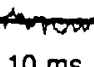

Figure 9. Fits of the voltage-offset model to the peak $G(V)$ and prepulse inactivation curves. $A$, Normalized peak conductance versus membrane voltage relation, $G(V)$ curve, from 4 whole-cell currents from $S h^{5}$ myotubes. The $G(V)$ curve was determined by dividing $I(V)$ curves like in Figure $1 C$ by the unitary current through single $A_{1}$ channels and normalizing to asymptote to 1 at high voltages. The smooth curves superimposed on the data are the $G(V)$ curves fitted to wild-type data (Zagotta and Aldrich, 1989) with and without a $20 \mathrm{mV}$ depolarizing shift. $B$, Prepulse inactivation curve from from 6 whole-cell currents from $S h^{5}$ myotubes. The prepulse inactivation curve was calculated as in Figure $2 C$. The smooth curve is the equilibrium probability of being not inactivated calculated from the model in Zagotta and Aldrich (1989) with and without a $20 \mathrm{mV}$ depolarizing shift. 


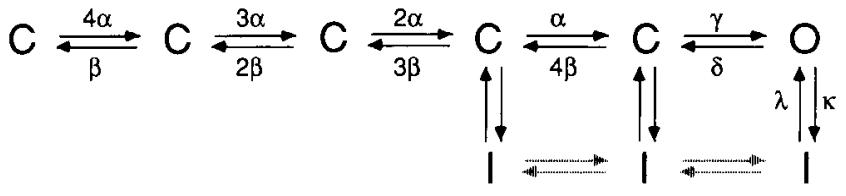

Figure 10. Summary of the model developed for the wild-type $A_{1}$ channel. The hatched arrows indicate transitions which may occur but are not addressed by this study.

(Zagotta and Aldrich, 1989). In short, the prepulse inactivation curve was fitted with an equilibrium formulation of the model (Zagotta and Aldrich, 1989, Eq. 4). From these fits we determined the equilibrium constant for the activation transition of each subunit at $0 \mathrm{mV}\left(A_{\alpha} / A_{\beta}\right)$ and the voltage dependence of the equilibrium $\left(1 / V_{\alpha}+1 / V_{\beta}\right)$. Next, the first latency distributions were fitted with numerical solutions of the model to determine the absolute magnitudes and voltage dependence of the activation $(\alpha)$ and deactivation $(\beta)$ rates. Finally, numerical solutions of the model were used to calculate macroscopic currents and peak $G(V)$ curves and compared to the corresponding data from $S h^{5} A_{1}$ channels.

A simple offset of the transmembrane voltage would predict both a decrease in $A_{u}$ and an increase in $\mathrm{A}_{\beta}$ without any change in either $V_{\alpha}$ or $V_{\theta}$. To produce a more depolarized, less steep prepulse inactivation curve in the model, the value of $A_{\alpha} / A_{\beta}$ must be decreased and the value of $\left(1 / V_{\alpha}+1 / V_{\beta}\right)$ must be decreased. However, the time course and voltage dependence of the first latency distributions are completely dominated at high voltages ( $>0 \mathrm{mV}$ for the wild-type model) by the amplitude and voltage dependence of $\alpha\left(A_{\alpha}\right.$ and $\left.V_{\alpha}\right)$. Since the first latencies of the $S h^{5} A_{1}$ channel were similar to wild-type latencies at +50 $\mathrm{mV}, \alpha$ is probably not appreciably affected by the mutation. Taken together, these results indicate that the primary effect of $S h^{5}$ is likely to be on the amplitude and voltage dependence of $\beta\left(A_{\beta}\right.$ and $\left.V_{\beta}\right)$.

Figure 11 replots the whole-cell $G(V)$ and prepulse inacti-

A

Peak Conductance vs Voltage

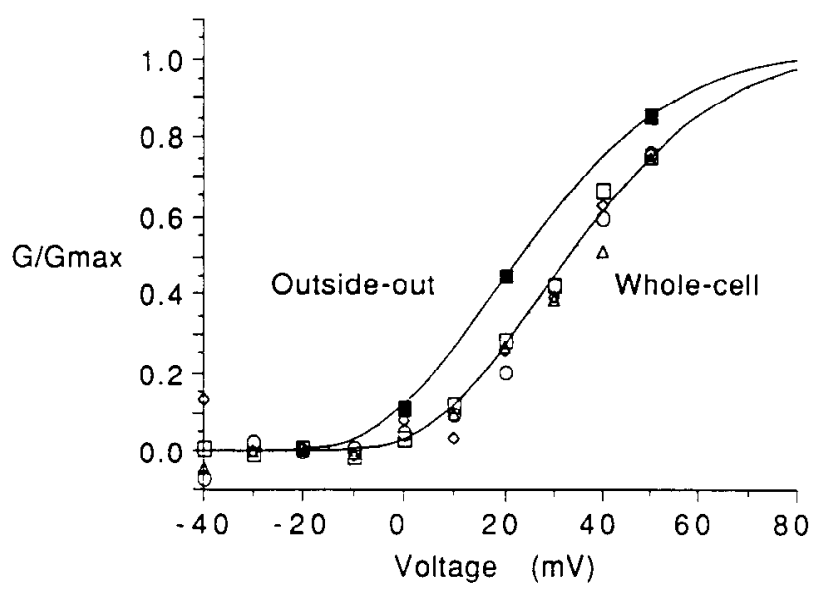

vation data of Figure 9, this time fitted with a modification of the wild-type model, where only the amplitude and voltage dependence of $\beta$ have been altered. The $\beta$-modified model quantitatively predicts both the depolarizing shifts and slope changes of the $G(V)$ and prepulse inactivation curves. Whereas the rate constant $\beta$ depends less steeply on voltage in the $\beta$-modified model relative to the wild-type model, the peak $G(V)$ curve of the $\beta$-modified model is steeper over most of the activation voltage range. This unexpected finding is only true at voltages where the currents are significantly activated $\left(G / G_{\max }>0.01\right)$; the foot of the peak $G(V)$ curve of the $\beta$-modified model, where the channels are just beginning to activate $\left(G / G_{\max }<0.01\right)$, is less steep as expected. The increased steepness at the higher voltages results from a differential effect of inactivation on the voltage dependence of the peak currents in the 2 models. When the inactivation transition is eliminated from the model, the slope of the $G(V)$ curve of the $\beta$-modified model is less than that of the wild-type model at all voltages. Peak $G(V)$ curves calculated with a rapid inactivation transition are significantly less steep at higher voltages and reach a limiting steepness only at very low voltages, making it virtually impossible to measure the limiting steepness experimentally.

Figure $11 A$ also plots the peak $G(V)$ data from the ensemble averages of Figure 3. It is clear from a comparison of the wholecell and single-channel data that a voltage offset exists between these 2 configurations. In a comparison of the sodium currents of outside-out patches and whole-cell membranes, Fernandez et al. (1984) have also observed a consistent depolarizing voltage offset in their whole-cell recordings. They attribute the offset to a Donnan potential in their whole-cell recording due to negatively charged macromolecules in the cell. We have found that, to produce adequate fits of the model to all of the whole-cell and single-channel data, we must consider the voltage of the whole-cell recordings to be offset by $10 \mathrm{mV}$ in the depolarizing direction.

Figure $12 A$ shows fits of the $\beta$-modified model to the first latency distributions of the $S h^{5} A_{1}$ channel at $0,+20$, and +50

B Prepulse Inactivation

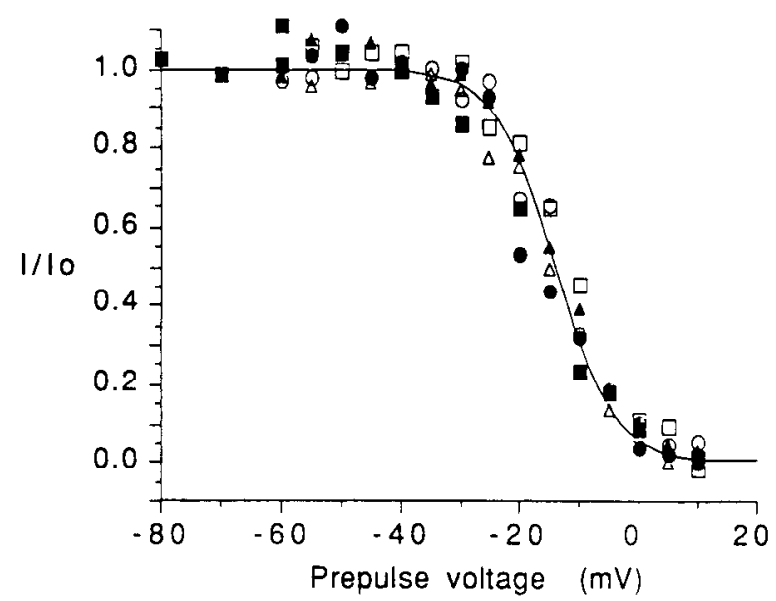

Figure 11. Fits of the $\beta$-modified model to the peak $G(V)$ and prepulse inactivation curves. $A$, The $S h^{5}$ whole-cell $G(V)$ data $(o p e n$ symbols) from Figure $9 A$ and single-channel $G(V)$ data (filled symbols) from the ensemble averages in Figure 3 are plotted with the predictions of the $\beta$-modified model. The model fit to the whole-cell data has been shifted $10 \mathrm{mV}$ in the depolarizing direction to account for the voltage offset of the whole-cell recordings. $B$, The $S h^{5}$ prepulse inactivation data from Figure $9 B$ are plotted with the predictions of the $\beta$-modified model shifted to account for the voltage offset of the whole-cell recordings. 


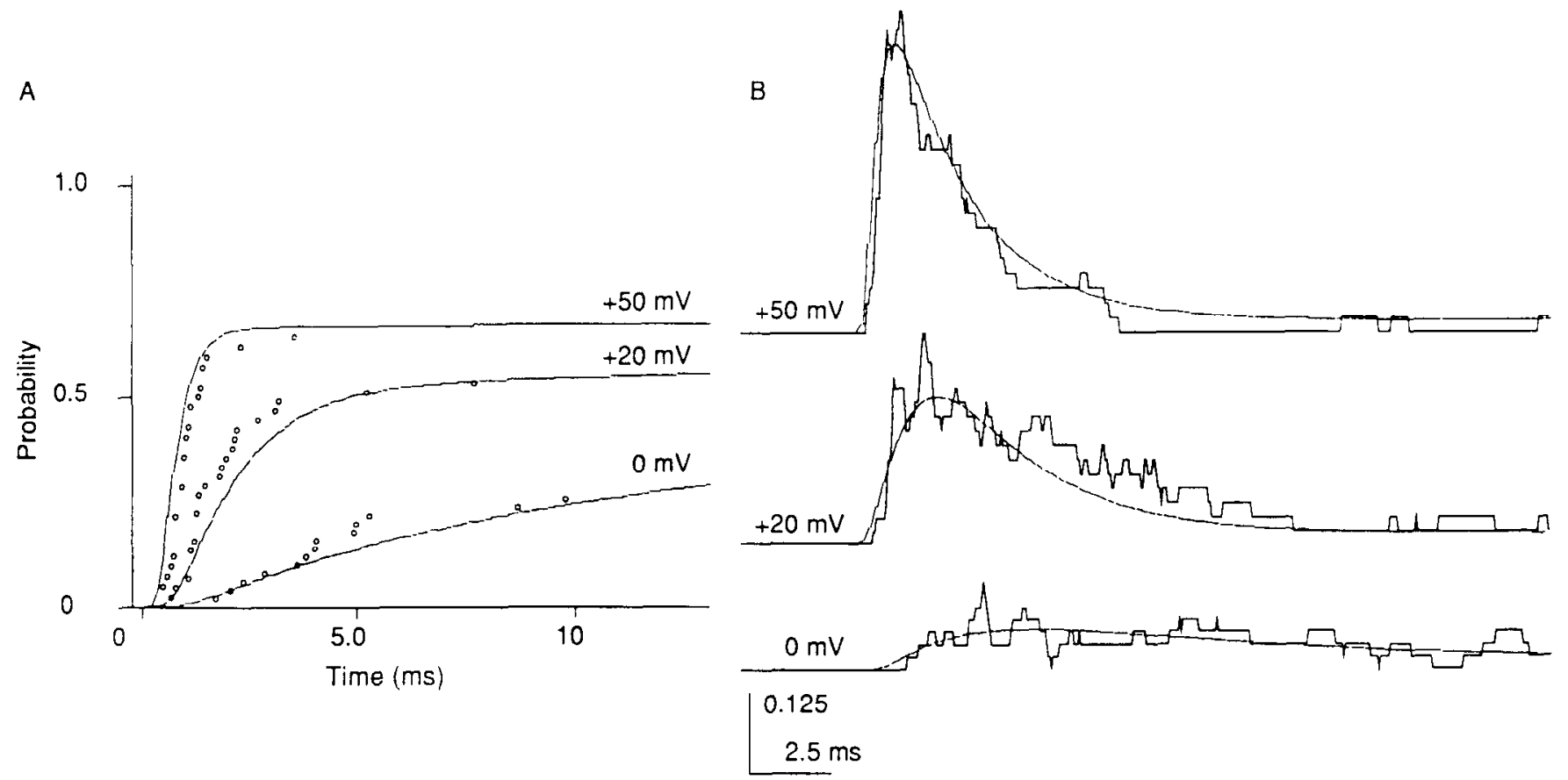

Figure 12. Fits of the $\beta$-modified model to first latencies and ensemble averages from a $S h^{5}$ single-channel outside-out patch. $A$, First latency distributions at $+50,+20$, and $0 \mathrm{mV}$ shown as dots fitted by the distributions calculated numerically from the $\beta$-modified model. $B$, Ensemble a verages at $+50,+20$, and $0 \mathrm{mV}$ superimposed on the open probabilities calculated numerically from the $\beta$-modified model. For these calculations, the probability of the channel being available to open was set to 0.74 to account for the occurrence of blank records at $+50 \mathrm{mV}$ in this patch. These blank records result from inactivation from closed states (Zagotta and Aldrich, 1989).

$\mathrm{mV}$. As compared to the predictions of wild-type model shown in Figure $7 A$, the fits of the $\beta$-modified model to the $S h^{5}$ first latency data are significantly improved. At $0 \mathrm{mV}$, the $\beta$-modified model predicts slower first latencies than the wild-type model, while at $+50 \mathrm{mV}$, the predicted latencics are virtually identical. Figure $12 B$ shows that the $\beta$-modified model also accurately reproduces the probability of being open, $P(t)$, at $0,+20$, and $+50 \mathrm{mV}$ as measured by the ensemble average of a single $S h^{5}$ $A_{1}$ channel.

The values of the rate constants used in the fits are summarized in Figure 13. The voltage dependence of the activation rate constants ( $\alpha$ and $\beta$ ) is ploted in Figure $13 B$ and expressed as an equation in Figure $13 C$. The model is identical to the wildtype model of Zagotta and Aldrich (1989) except for the values for the amplitude and voltage dependence of $\beta$. The new value of $V_{B}$ corresponds to a reduction in the charge movement relative to wild-type of approximately 0.6 charges per subunit between the open state of the subunit and the transition state for closing. The charge movement between the closed state of the subunit and the transition state for opening is not significantly altered by the $S h^{i}$ mutation. Differences in the gating of the wild-type and $S h^{5} \mathrm{~A}$-channels at $0 \mathrm{mV}$ indicate that the voltage-independent component of the rate constant $\left(\mathrm{A}_{i}\right)$ must also be altered by the mutation. The new value of $\mathrm{A}_{\beta}$ corresponds to an energy difference at $0 \mathrm{mV}$ of $12.2 \mathrm{kcal} / \mathrm{mol}$ between the open state of the subunit and the transition state to closing, consistent with a destabilization of the open state of the subunit of $1.2 \mathrm{kcal} /$ mol for the $S h^{s} \mathrm{~A}_{1}$ channel relative to the wild-type channel.

\section{Discussion}

We have analyzed the alterations in gating of single A-type potassium channels produced by a mutation in a structural gene for a component of the channel, $S h^{5}$. The studies were carried out on cultured embryonic myotubes, for which we have previously reported a detailed kinetic analysis of the single wildtype channels from the $S h$ locus (Zagotta and Aldrich, 1989). The gating of the $S h^{5}$ channels was shown to be altered in the following ways. (1) The activation of the channel occurs at a more depolarized voltage, producing a peak $G(V)$ curve with a steeper slope. (2) The prepulse inactivation also occurs at more depolarized voltages and depends less steeply on voltage. (3) The first latencies are slower at the lower voltages $(<20 \mathrm{mV})$. The voltage-independent transition rates, into and out of the open state, were not significantly affected by the mutation. While we cannot totally eliminate the contribution of the delayed currents to the whole-cell data, it seems likely that these alterations are not due to a simple voltage shift, as would be expected if the mutation resulted in a change in the surface charge of the protein, producing an offset of the transmembrane voltage sensed by the channel. By fitting the macroscopic and single-channel data with the model developed for the wild-type channel, we show that all of these alterations in voltage-dependent kinetics can be accounted for by altering only the deactivation or closing rate constant, $\beta$, for each subunit. To reproduce the depolarizing shifts of the $G(V)$ and prepulse inactivation curves and the increased latencies at low voltages, the amplitude of $\beta, A_{\beta}$, had to be increased, and to reproduce the decrease in slope of the prepulse inactivation curve, the voltage dependence of $\beta$ had to be decreased (increasing the parameter $V_{\beta}$ ).

These alterations in $\beta$ provide insights into how the structural change produced by the $S h^{5}$ mutation has altered the channel's behavior. The increased value of $A_{\beta}$ suggests a destabilization of the open conformation of each subunit, and the increased value of $V_{\beta}$ suggests a decreased charge movement in the mem- 
A
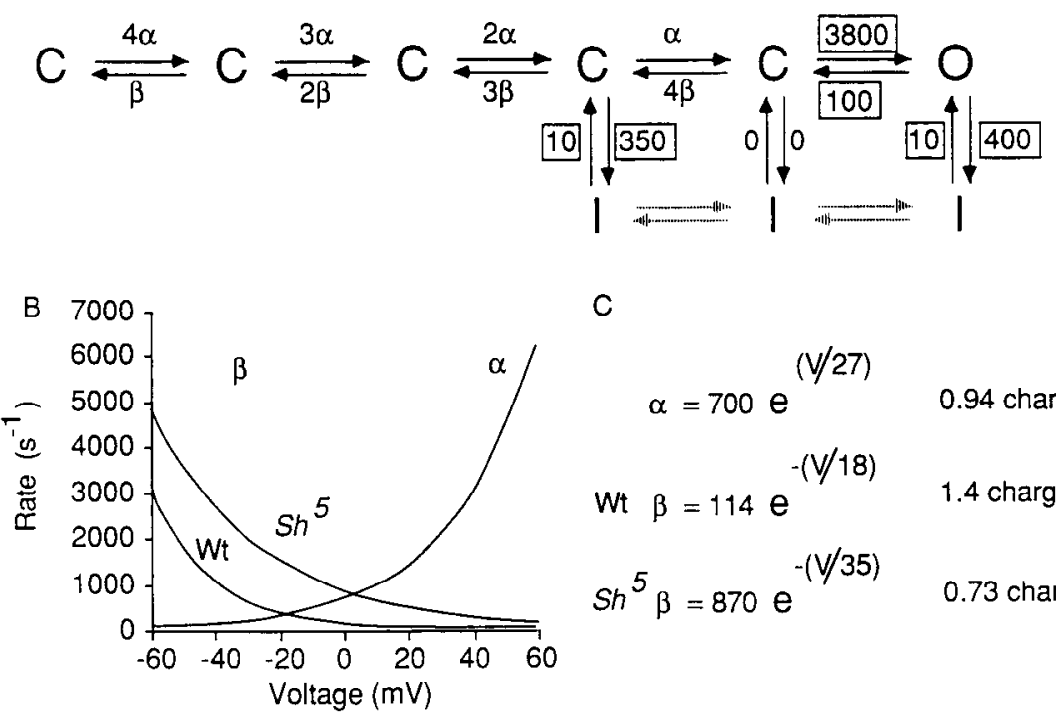

C

$$
\begin{aligned}
& \alpha=700 e^{(V / 27)} \quad 0.94 \text { charges } \\
& \text { Wt } \beta=114 e^{-(V / 18)} \quad 1.4 \text { charges } \\
& S h^{5} \beta=870 e^{-(V / 35)} \quad 0.73 \text { charges }
\end{aligned}
$$

Figure 13. Summary of the rates used in the $\beta$-modified model. $A$, State diagram of the model showing the absolute magnitudes of the voltage-independent rates (per sec) and the relative magnitudes of the voltage-dependent rates. The rate constants that do not depend on voltage are boxed. These voltageindependent rates are identical to the rates of the wild-type model $(B)$. Exponential voltage dependence of the activation and deactivation rates showing both the wild-type and $S h^{5} \beta(V)$ function. $C$, Functional forms of the activation and deactivation rates showing the equivalent charge movement associated with these rates. brane field associated with the closing conformational change. One way of producing both of these effects is to mutate a structure closely associated with the movement of gating charge during the activation process. Since the positively charged residues in $S 4$ are thought to sense the change in transmembrane field and move in response to it, a mutation that interferes with this mechanism could reduce the amount of charge movement as well as destabilize the open conformation. In sodium channels, neutralization of $\mathrm{S} 4$ charges produces a decreased slope of the voltage dependence of steady-state activation $\left(m_{\infty}{ }^{3}\right)$, which is frequently associated with a depolarizing shift (Stühmer et al., 1989). If the opening conformational change of each subunit was prevented from occurring to completion by the $S h^{5}$ mutation, but still occurred far enough to allow the channel to open, the open subunits would close more rapidly and with less associated charged movement.

Recently, an alteration was found in the primary structure of the $S h$ channel from the $S h^{5}$ allele (Gautam and Tanouye, unpublished observations). A phenylalanine has been changed to a isoleucine in the region between the $S 4$ sequence and the next proposed transmembrane segment (GLLIFFL $\rightarrow$ GLLIIFL). The proximity of this mutation to the $\mathrm{S} 4$ region raises the possibility that it could influence coupling of sliding of the S4 helix to activation of the subunit, as described. McCormack et al. (1989) have reported the finding of a leucine zipper motif in the $S h$ channel near the $S 4$ sequence, in the region of the $S h^{5}$ mutation. This motif is conserved in most other voltage-gated channels and was suggested to play a role in communicating the movement of the S4 helix to the rest of the channel molecule. The finding that the mutation does not alter the charged character of the amino acid is consistent with our finding that the altered function is not likely to result from a simple offset of the transmembrane voltage sensed by the channel.

Alternative splicing of the $S h$ locus raises the possibility that the altered gating in $S h^{5}$ is an indirect result of the mutation eliminating a subset of the variants rather than altering the structure of the variant(s) normally expressed. The remaining variants might then combine to form a channel with different voltage-dependent kinetics. We believe that this mechanism is not a likely origin for the altered channel function. In contrast to the effects of $S h^{5}$, the different $S h$ variants, when expressed in Xenopus oocytes, produce channels with similar voltage dependence, differing primarily in their inactivation rate and rate of recovery from inactivation (Iverson et al., 1988; Timpe et al., 1988a, b). ShB and ShD variants, differing only in the amino terminal cytoplasmic region, have quantitatively similar first latencies but differ significantly in their open durations and the number of openings per burst (Zagotta et al., 1989a). This is consistent with the $S h^{5}$ mutation residing in the constant region of the $S / h$ polypeptide.

Previous voltage-clamp experiments suggest that alterations in $S h^{5} \mathrm{~A}$-currents may differ for different developmental stages or different tissues. The A-currents in dorsal longitudinal flight muscles (DLMs) of $S h^{5}$ pupae exhibit abnormally fast macroscopic inactivation and recovery from inactivation (Salkoff and Wyman, 1981b), more like the currents from the different $S h$ variants in Xenopus oocytes. In contrast, the A-current in $S h^{5}$ larval body wall muscles show a voltage shift of both activation and inactivation processes (Wu and Haugland, 1985), similar to the effects of $S h^{5}$ on the embryonic myotubes. Since the embryonic muscles are progenitors to the larval muscles, the $S h$ effect does not change significantly as these muscles develop. However, the adult DLMs are generated de novo during pupation. The differences in the effects of $S h^{5}$ in the pupal DLMs may indicate the expression of different $S h$ variants at this new developmental stage and differences in the effects of the $S h^{5}$ mutation on the different variants.

The effects of $S h^{5}$ on both activation and inactivation provide further support that these processes are intimately coupled. Since the channels generally open before they inactivate, an alteration in the processes leading to opening will affect the inactivation of the channel as well. Moreover, the manner in which the activation is altered provides insights into the role of the mutated structure in the gating mechanism. 


\section{References}

Aldrich RW, Stevens CF (1987) Voltage-dependent gating of single sodium channels from mammalian neuroblastoma cells. J Neurosci $7: 418-431$.

Aldrich RW, Corey DP, Stevens CF (1983) A reinterpretation of mammalian sodium channel gating based on single-channel recording. Nature 306:436-441.

Armstrong CM, Bezanilla F (1977.) Inactivation of the sodium channel. II. Gating current experiments. J Gen Physiol. 70:567-590.

Baumann A, Krah-Jentgens I, Muller R, Muller-Holtkamp F, Seidel R, Kecskemethy N, Casal J, Ferrus A, Pongs O (1987) Molecular organization of the maternal effect region of the Shaker complex of Drosophila: characterization of an $\mathrm{I}_{\mathrm{A}}$ channel transcript with homology to vertebrate $\mathrm{Na}+$ channel. EMBO J 6:3419-3429.

Baumann A, Grupe A, Ackermann A, Pongs O (1988) Structure of the voltage-dependent potassium channel is highly conserved from Drosophila to vertebrate central nervous systems. EMBO J 7:24572463.

Catterall WA (1986) Voltage-dependent gating of sodium channels: correlating structure and function. Trends Neurosci 9:7-10.

Ellis SB, Williams ME, Ways NR, Brenner R, Sharp AH, Leung AT, Campbcll KP, McKenna E, Koch WJ, Hui A, Schwartz A, Harpold MM (1988) Sequence and expression of mRNAs encoding the $a_{1}$ and a, subunits of a DHP-sensitive calcium channel. Nature 241: 1661-1664.

Fernandez JM, Fox AP, Krasne S (1984) Membrane patches and whole-cell membranes: a comparison of electrical properties in rat clonal pituitary $\left(\mathrm{GH}_{3}\right)$ cells. J Physiol (Lond) 356:565-585.

Greenblatt RE, Blatt Y, Montal M (1985) The structure of the voltagesensitive sodium channel. Inferences derived from computer-aided analysis of the Electrophorus electricus channel primary structure. FEBS Lett 193:125-134.

Guy HR, Seetharamulu P (1986) Molecular model of the action potential sodium channel. Proc Natl Acad Sci USA 83:508-512.

Iverson LE, Tanouye MA, Lester HA, Davidson N, Rudy B (1988) A-typc potassium channels expressed from Shaker locus cDNA. Proc Natl Acad Sci USA 85:5723-5727.

Jan YN, Jan LY, Dennis MJ (1977) Two mutations of synaptic transmission in Drosophila. Proc R Soc Lond [Biol] 198:87-108.

Kamb A, Iverson LE, Tanouye MA (1987) Molecular characterization of Shaker, a Drosophila gene that encodes a potassium channel. Cell 50:405-413.

Kamb A, Tseng-Crank J, Tanouye MA (1988) Multiple products of the Drosophila Shaker gene may contribute to potassium channel diversity. Neuron 1:421-430.

Kaplan WD, Trout WE (1969) The behavior of four neurological mutants of Drosophila. Genetics 61:399-409.

Kayano T, Noda M, Flockerzi V, Takahashi H, Numa S (1988) Primary structure of rat brain sodium channel III deduced from the cDNA sequence. FEBS Lett 228:187-194.

Loughney K, Kreber R, Ganetzky B (1989) Molecular analysis of the para locus, a sodium channel gene in Drosophila. Cell 58:1143-1154.

McCormack K, Campanelli JT, Ramaswami M, Mathew MK, Tanouye MA, Iverson LE, Rudy B (1989) Leucine-zipper motif update. Nature 340:103.

Noda M, Shimizu S, Tanabe T, Takai T, Kayano T, Ikeda T, Takahashi H, Nakayama H, Kanaoka Y, Minamino N, Kangawa K, Matsuo H, Raftery MA, Hirose T, Inayama S, Hayashida $H$, Miyata $T$, Numa S (1984) Primary structure of Electrophorus electricus sodium channel deduced from cDNA sequence. Nature 312:121-127.

Noda M, Ikeda T, Kayano T, Suzuki H, Takeshima H, Kurasaki M, Takahashi H, Numa S (1986) Existence of distinct sodium channel messenger RNAs in rat brain. Nature 320:188-192.

Papazian DM, Schwarz TL, Tempel BL, Jan YN, Jan LY (1987) Cloning of genomic and complementary DNA from Shaker, a putative potassium channel gene from Drosophila. Science 237:749-753.

Pongs O, Kecskemethy N, Muller R, Kreh-Jentgens I, Baumann A, Kiltz HH, Canal I, Llamazares S, Ferrus A (1988) Shaker encodes a family of putative potassium channel proteins in the nervous system of Drosophila. EMBO J 7:1087-1096.

Salkoff LB (1983a) Drosophila mutants reveal two components of fast outward current. Nature 302:249-251.

Salkoff LB (1983b) Genetic and voltage-clamp analysis of a Drosophila potassium channel. Cold Spring Harbor Symp Quant Biol. 48:221231.

Salkoff LB, Wyman R (1981a) Outward currents in developing Drosophila flight muscle. Science 212:461-463.

Salkoff LB, Wyman R (1981b) Genetic modification of potassium channels in Drosophila Shaker mutants. Nature 293:228-230.

Salkoff LB, Wyman R (1983) Ion currents in Drosophila flight muscles J Physiol 337:687-709.

Salkoff L, Butler A, Wei A, Scavarda N, Giffen K, Ifune C, Goodman R, Mandel G (1987) Genomic organization and deduced amino acid sequence of a putative sodium channel gene in Drosophila. Science 237:744-749.

Schwarz TL, Tempel BL, Papazian DM, Jan YN, Jan LY (1988) Multiple potassium-channel components are produced by alternative spicing at the Shaker locus of Drosophila. Nature 331:137-142.

Solc CK, Aldrich RW (1988) Voltage-gated potassium channels in larval CNS neurons of Drosophila. J Neurosci 8:2556-2570.

Solc CK, Zagotta WN, Aldrich RW (1987) Single-channel and genetic analyses reveal two distinct A-type potassium channels in Drosophila. Science 236:1094-1098.

Stühmer W, Conti F, Suzuki H, Wang X, Noda M, Yahagi N, Kubo $H$, Numa $S$ (1989) Structural parts involved in activation and inactivation of the sodium channel. Nature 339:597-603.

Tanabe T, Takeshima H, Mikami A, Flockerzi V, Takahashi H, Kangawa K, Kojima M, Matsuo H, Hirose T, Numa S (1987) Primary structure of the receptor for calcium channel blockers from skeletal muscle. Nature 328:313-318.

Tanabe T, Beam KG, Powell JA, Numa S (1988) Restoration of excitation-contraction coupling and slow calcium current in dysgenic muscle by dihydropyridine receptor complementary DNA. Nature 336:134-139.

Tempel BL, Papazian DM, Schwarz TL, Jan YN, Jan LY (1987) Sequence of a probable potassium channel component encoded at Shaker locus of Drosophila. Science 237:749-753.

Tempel BL, Jan YN, Jan LY (1988) Cloning of a probable potassium channel gene from mouse brain. Nature 332:837-839.

Timpe LC, Jan LY (1987) Gene dosage and complementation analysis of the Shaker locus in Drosophila. J Neurosci 7:1307-1317.

Timpe LC, Schwarz TL, Tempel BL, Papazian DM, Jan YN, Jan LY (1988a) Expression of functional potassium channels from Shaker cDNA in Xenopus oocytes. Nature 331:143-145.

Timpe LC, Jan YN, Jan LY (1988b) Four cDNA clones from the Shaker locus of Drosophila induce kinetically distinct A-type potassium currents in Xenopus oocytes. Neuron 1:659-667.

Wu C-F, Haugland FN (1985) Voltage clamp analysis of membrane currents in larval muscle fibers of Drosophila: alteration of potassium currents in Shaker mutants. J Neurosci 5:2626-2640.

Wu C-F, Ganetzky B, Haugland FN, Liu AX (1983) Potassium currents in Drosophila: different components affected by mutations of two genes. Science 220:1076-1078.

Zagotta WN, Aldrich RW (1989) Voltage-dependent gating of Shaker A-type potassium channels in Drosophila muscle. J Gen Physiol 95: 29-60.

Zagotta WN, Brainard MS, Aldrich RW (1988) Single-channel analysis of four distinct classes of potassium channels in Drosophila muscle. J Neurosci 8:4765-4779.

Zagotta WN, Hoshi T, Aldrich RW (1989a) Gating of single Shaker $\mathrm{K}^{+}$channels in Drosophila muscle and in Xenopus oocytes injected with Shaker mRNA. Proc Natl Acad Sci USA 86:7243-7247.

Zagotta WN, Germeraad S, Garber SS, Hoshi T, Aldrich RW (1989b) Properties of ShB A-type $\mathrm{K}^{+}$channels expressed in Shaker mutant Drosophila by germline transformation. Neuron 3:773-782. 